\title{
THE 3G INEQUALITY FOR A UNIFORMLY JOHN DOMAIN
}

\author{
Hiroaki Aikawa and TorbJörn LundH \\ Dedicated to the memory of Professor Nobuyuki Suita
}

\begin{abstract}
Let $G$ be the Green function for a domain $D \subset \mathbf{R}^{d}$ with $d \geq 3$. The Martin boundary of $D$ and the $3 \mathrm{G}$ inequality:

$$
\frac{G(x, y) G(y, z)}{G(x, z)} \leq A\left(|x-y|^{2-d}+|y-z|^{2-d}\right) \quad \text { for } x, y, z \in D
$$

are studied. We give the $3 \mathrm{G}$ inequality for a bounded uniformly John domain $D$, although the Martin boundary of $D$ need not coincide with the Euclidean boundary. On the other hand, we construct a bounded domain such that the Martin boundary coincides with the Euclidean boundary and yet the $3 \mathrm{G}$ inequality does not hold.
\end{abstract}

\section{Introduction}

For a bounded Lipschitz domain $D \subset \mathbf{R}^{d}$ with $d \geq 3$, Cranston, Fabes and Zhao [13] proved the following $3 \mathrm{G}$ inequality:

$$
\frac{G(x, y) G(y, z)}{G(x, z)} \leq A_{0}\left(|x-y|^{2-d}+|y-z|^{2-d}\right) \quad \text { for } x, y, z \in D
$$

where $G$ is the Green function for $D$ and $A_{0}$ is a positive constant depending only on $D$. They used (1) for the conditional gauge theorem and the Schrödinger equation. Their proof is based on the boundary Harnack principle, a comparison principle among positive harmonic functions vanishing on a portion of the boundary $([6,15,18])$. The boundary Harnack principle also yields the coincidence of the Martin boundary of $D$ and the Euclidean boundary ([16]). So, one might think that there is a relationship between the $3 \mathrm{G}$ inequality and the

2000 Mathematics Subject Classification. 31B05, 31B25.

Key words and phrases. Green function, 3G inequality, boundary Harnack principle, uniformly John domain, inner uniform domain.

This work was supported in part by Grant-in-Aid for Scientific Research (B) (No. 15340046) and Exploratory Research (No. 13874023) Japan Society for the Promotion of Science.

Received March 9, 2004; revised September 6, 2004. 
coincidence of the Martin and the Euclidean boundaries. We shall however see that there is no direct connection between them. We shall prove the $3 \mathrm{G}$ inequality for a uniformly John domain, whose Martin boundary need not coincide with the Euclidean boundary (Theorem 1). On the other hand, we shall provide an example of a bounded domain in $\mathbf{R}^{d}$ with $d \geq 3$, whose Martin boundary coincides with the Euclidean boundary and for which the $3 \mathrm{G}$ inequality fails to hold (Proposition 2).

Throughout the paper, let $D$ be a bounded domain in $\mathbf{R}^{d}, d \geq 3$, and let $\delta_{D}(x)=\operatorname{dist}(x, \partial D)$. For $x, y \in D$, we define the internal metric or the inner diameter distance $\rho_{D}(x, y)$ by

$$
\rho_{D}(x, y)=\inf \{\operatorname{diam}(\gamma)\}
$$

where the infimum is taken over all curves $\gamma$ connecting $x$ and $y$ in $D$ and $\operatorname{diam}(\gamma)$ stands for the diameter of $\gamma$. The inner length distance $\lambda_{D}(x, y)$ is defined similarly by $\lambda_{D}(x, y)=\inf \{\ell(\gamma)\}$, where the infimum is taken over all curves $\gamma$ connecting $x$ and $y$ in $D$ and $\ell(\gamma)$ stands for the length of $\gamma$. Obviously $|x-y| \leq \rho_{D}(x, y) \leq \lambda_{D}(x, y)$. If $|x-y| \leq \max \left\{\delta_{D}(x), \delta_{D}(y)\right\}$, then $|x-y|=$ $\rho_{D}(x, y)=\lambda_{D}(x, y)$. We say that $D$ is a uniformly John domain if there exists a constant $A_{1} \geq 1$ such that each pair of points $x, y \in D$ can be connected by a curve $\gamma \subset D$ for which

$$
\begin{aligned}
& \min \{|x-z|,|z-y|\} \leq A_{1} \delta_{D}(z) \quad \text { for all } z \in \gamma, \\
& \operatorname{diam}(\gamma) \leq A_{1} \rho_{D}(x, y)
\end{aligned}
$$

(Balogh and Volberg $[7,8]$ ). We say that $D$ is an inner uniform domain if there exists a constant $A_{2} \geq 1$ such that each pair of points $x, y \in D$ can be connected by a curve $\gamma \subset D$ for which

$$
\begin{aligned}
& \min \{\ell(\gamma(x, z)), \ell(\gamma(z, y))\} \leq A_{2} \delta_{D}(z) \text { for all } z \in \gamma, \\
& \ell(\gamma) \leq A_{2} \lambda_{D}(x, y),
\end{aligned}
$$

where $\gamma(x, z)$ is the subarc of $\gamma$ connecting $x$ and $z$ (Bonk, Heinonen and Koskela [11]). In view of Väisälä [17], the family of uniformly John domains and that of inner uniform domains coincide.

Under some additional assumptions, such as the the uniform perfectness of the boundary or the existence of a strong barrier, Balogh-Volberg and BonkHeinonen-Koskela studied the boundary Harnack principle and the Martin boundary for these domains. In [4] Mizutani and the authors gave the boundary Harnack principle and identified the Martin boundary of a bounded uniformly John domain without any other additional assumptions. The Martin boundary is the ideal boundary with respect to the internal metric $\rho_{D}(x, y)$; it need not be homeomorphic to the Euclidean boundary. In this note we show the following.

THEOREM 1. Let $D$ be a bounded uniformly John domain in $\mathbf{R}^{d}$ with $d \geq 3$. Then the $3 G$ inequality (1) holds. 
In Section 3 we shall construct a bounded domain in $\mathbf{R}^{d}$ with $d \geq 3$, such that the Martin boundary coincides with the Euclidean boundary and yet the $3 \mathrm{G}$ inequality does not hold.

Remark 1. There is a significant difference between the planar case and the case $d \geq 3$. For the planar case Bass and Burdzy [10] established the 3G inequality (with suitable modification of the right hand side) for an arbitrary bounded domain.

\section{Proof of Theorem 1}

We shall use the following notation as in [4]. By the symbol $A$ we denote a positive constant depending only on the dimension $d$, whose value is unimportant and may change even in the same line. We shall say that two positive functions $f_{1}$ and $f_{2}$ are comparable, written $f_{1} \approx f_{2}$, if and only if there exists a constant $A \geq 1$ such that $A^{-1} f_{1} \leq f_{2} \leq A f_{1}$. The constant $A$ will be called the constant of comparison. By $B(x, r)$ we denote the open ball with center at $x$ and radius $r$.

Let $D^{*}$ be the completion of $D$ with respect to the internal metric. That is, $D^{*}$ is the equivalence class of all $\rho_{D^{-}}$-Cauchy sequences with equivalence relation " $\sim$ ", where we say $\left\{x_{j}\right\} \sim\left\{y_{j}\right\}$ if $\left\{x_{j}\right\} \cup\left\{y_{j}\right\}$ is a $\rho_{D}$-Cauchy sequence. The completion $D^{*}$ is a compact space. Let $\partial^{*} D=D^{*} \backslash D$, the boundary with respect to $\rho_{D}$. Take $\xi^{*} \in D^{*}$. Suppose $\xi^{*}$ is represented by a $\rho_{D}$-Cauchy sequence $\left\{x_{j}\right\}$. Since $\left\{x_{j}\right\}$ is also a usual Cauchy sequence, it follows that $x_{j}$ converges to some point $\xi \in \bar{D}$. The point $\xi$ is independent of the representative sequence $\left\{x_{j}\right\}$ and uniquely determined by $\xi^{*}$. We say that $\xi^{*}$ lies over $\xi \in \bar{D}$. If $\xi \in D$, then $\xi$ and $\xi^{*}$ coincide. Define the projection $\pi: D^{*} \rightarrow \bar{D}$ by $\pi\left(\xi^{*}\right)=\xi$. Let $B_{\rho}\left(\xi^{*}, r\right)$ be the open connected component of $D \cap B\left(\pi\left(\xi^{*}\right), r\right)$ which can be connected to $\xi^{*}$ in itself, i.e. for every $x \in B_{\rho}\left(\xi^{*}, r\right)$ there is an arc $\gamma \subset B_{\rho}\left(\xi^{*}, r\right)$ starting from $x$ and converging to $\xi^{*}$. By definition $\rho_{D}\left(\xi^{*}, x\right)<2 r$ for $x \in B_{\rho}\left(\xi^{*}, r\right)$; in other words

$$
\text { if } \rho_{D}\left(\xi^{*}, x\right) \geq 2 r, \text { then } x \in D \backslash B_{\rho}\left(\xi^{*}, r\right) \text {. }
$$

Let $\xi^{*} \in \partial^{*} D$. Observe from (2) that

(4) if there exists $y \in D$ with $\rho_{D}\left(\xi^{*}, y\right) \geq 2 r$, then there exists $x \in B_{\rho}\left(\xi^{*}, r\right)$ with $\delta_{D}(x) \approx r$.

In [4] Mizutani and the authors proved the following.

Theorem A. Let $D$ be a bounded uniformly John domain. Then the Martin compactification of $D$ is homeomorphic to $D^{*}$ and each boundary point $\xi^{*} \in \partial^{*} D$ is minimal.

This theorem was deduced as a corollary to a uniform boundary Harnack principle, whose proof is based on the following estimate of the Green function (cf. [3, Lemma 3] and [4, Lemma 3.2]). 
Lemma A. Let $\xi^{*} \in \partial^{*} D$. Then

$$
\frac{G(x, y)}{G\left(x^{\prime}, y\right)} \approx \frac{G\left(x, y^{\prime}\right)}{G\left(x^{\prime}, y^{\prime}\right)} \quad \text { for } x, x^{\prime} \in B_{\rho}\left(\xi^{*}, r\right) \text { and } y, y^{\prime} \in D \backslash B_{\rho}\left(\xi^{*}, 6 r\right)
$$

with constant comparison depending only on $D$.

In [4, Lemma 3.2], the above estimate was given actually for the Green function for the intersection of $D$ and $B_{\rho}\left(\xi^{*}, A r\right)$ with $A$ large enough. However, for the case $d \geq 3$, we see that the same estimate holds for the Green function for $D$ itself.

We also need the following lemma whose proof is easy and left to the reader.

Lemma 1. Let $x, y \in D$. Then $G(x, y) \leq A \rho_{D}(x, y)^{2-d}$. Moreover, if $\delta_{D}(x) \geq A^{-1} \rho_{D}(x, y)$ and $\delta_{D}(y) \geq A^{-1} \rho_{D}(x, y)$, then $G(x, y) \geq A^{-1} \rho_{D}(x, y)^{2-d}$.

Proof of Theorem 1. We have observed $|x-y| \leq \rho_{D}(x, y)$. So, let us prove the following slightly stronger form of the $3 \mathrm{G}$ inequality.

$$
\frac{G(x, y) G(y, z)}{G(x, z)} \leq A\left(\rho_{D}(x, y)^{2-d}+\rho_{D}(y, z)^{2-d}\right) \text { for } x, y, z \in D .
$$

We will prove (5) according to the line of Bass' proof of the $3 \mathrm{G}$ inequality. See [9, Theorem 3.6] and its correction. Let $c_{1}=\frac{1}{39}$ and $c_{2}=\frac{1}{13} c_{1}$. By symmetry we may assume that

$$
\rho_{D}(x, y) \leq \rho_{D}(y, z) .
$$

CASE 1. $\rho_{D}(x, y) \geq c_{1} \rho_{D}(x, z)$. Let $r=\rho_{D}(x, z)$. If $\delta_{D}(x) \geq c_{2} r$, then we let $x_{1}=x$. If $\delta_{D}(x)<c_{2} r$, then we take $x_{1}$ as follows: Let $x^{\prime} \in \partial D$ with $\left|x-x^{\prime}\right|=\delta_{D}(x)$. Since the line segment $\overline{x x^{\prime}}$ is included in $D \cap B\left(x^{\prime}, c_{2} r\right)$, we find $x^{*} \in \partial^{*} D$ lying over $x^{\prime}$ such that $x \in B_{\rho}\left(x^{*}, c_{2} r\right)$. Then

$$
\begin{aligned}
& \rho_{D}\left(x^{*}, y\right) \geq \rho_{D}(x, y)-\rho_{D}\left(x, x^{*}\right) \geq\left(c_{1}-c_{2}\right) r=12 c_{2} r, \\
& \rho_{D}\left(x^{*}, z\right) \geq \rho_{D}(x, z)-\rho_{D}\left(x, x^{*}\right) \geq\left(1-c_{2}\right) r>12 c_{2} r .
\end{aligned}
$$

By (4) we can take $x_{1} \in B_{\rho}\left(x^{*}, c_{2} r\right)$ with $\delta_{D}\left(x_{1}\right) \approx c_{2} r$. Then $x, x_{1} \in B_{\rho}\left(x^{*}, c_{2} r\right)$ and $y, z \in D \backslash B_{\rho}\left(x^{*}, 6 c_{2} r\right)$ by (3), so that Lemma A yields

$$
\frac{G(x, y)}{G\left(x_{1}, y\right)} \approx \frac{G(x, z)}{G\left(x_{1}, z\right)} \text {. }
$$

Similarly, if $\delta_{D}(z) \geq c_{2} r$, then we let $z_{1}=z$. If $\delta_{D}(z)<c_{2} r$, then we take $z^{\prime} \in \partial D$ with $\left|z-z^{\prime}\right|=\delta D(z)$ and $z^{*} \in \partial^{*} D$ lying over $z^{\prime}$ such that $z \in B_{\rho}\left(z^{*}, c_{2} r\right)$. By (6)

$$
\begin{aligned}
& \rho_{D}\left(z^{*}, y\right) \geq \rho_{D}(z, y)-\rho_{D}\left(z, z^{*}\right) \geq\left(c_{1}-c_{2}\right) r=12 c_{2} r, \\
& \rho_{D}\left(z^{*}, x_{1}\right) \geq \rho_{D}(x, z)-\rho_{D}\left(x, x_{1}\right)-\rho_{D}\left(z, z^{*}\right) \geq\left(1-2 c_{2}\right) r>12 c_{2} r .
\end{aligned}
$$


Hence we find $z_{1} \in B_{\rho}\left(z^{*}, c_{2} r\right)$ such that $\delta_{D}\left(z_{1}\right) \approx c_{2} r$ by (4). Then $z, z_{1} \in$ $B_{\rho}\left(z^{*}, c_{2} r\right)$ and $y, x_{1} \in D \backslash B_{\rho}\left(z^{*}, 6 c_{2} r\right)$, so that Lemma A yields

$$
\frac{G(y, z)}{G\left(x_{1}, z\right)} \approx \frac{G\left(y, z_{1}\right)}{G\left(x_{1}, z_{1}\right)} \text {. }
$$

Hence

$$
\frac{G(x, y) G(y, z)}{G(x, z)} \approx \frac{G\left(x_{1}, y\right) G\left(y, z_{1}\right)}{G\left(x_{1}, z_{1}\right)} .
$$

Now observe that $\delta_{D}\left(x_{1}\right) \approx \delta_{D}\left(z_{1}\right) \approx \rho_{D}\left(x_{1}, z_{1}\right) \approx r$, so that $G\left(x_{1}, z_{1}\right) \approx r^{2-d}$ by Lemma 1. Also, $\rho_{D}\left(x_{1}, y\right) \approx \rho_{D}(x, y) \geq c_{1} r$ and $\rho_{D}\left(y, z_{1}\right) \approx \rho_{D}(y, z) \geq \rho_{D}(x, y)$ by (6). Hence Lemma 1 yields

$$
\frac{G(x, y) G(y, z)}{G(x, z)} \leq A \frac{\rho_{D}(x, y)^{2(2-d)}}{r^{2-d}} \leq A \rho_{D}(x, y)^{2-d} .
$$

Thus (5) holds in this case.

CASE 2. $\rho_{D}(x, y)<c_{1} \rho_{D}(x, z)$. Let $s=\rho_{D}(x, y)$. By connectedness there is $w \in D$ with $\rho_{D}(x, w)=s / c_{1}$. Then

$$
\rho_{D}(y, w) \geq \rho_{D}(x, w)-\rho_{D}(x, y)=\left(\frac{1}{c_{1}}-1\right) s>s=\rho_{D}(x, y)=c_{1} \rho_{D}(x, w),
$$

so that Case 1 applies to the triplet $x, y, w$. Hence

$$
\frac{G(x, y) G(y, w)}{G(x, w)} \leq A \rho_{D}(x, y)^{2-d} .
$$

We are now going to replace $w$ with $z$.

SubCASE 2a. $s=\rho_{D}(x, y)>\frac{1}{2} \delta_{D}(y)$. Let $y^{\prime} \in \partial D$ with $\left|y-y^{\prime}\right|=\delta_{D}(y)$. Since the line segment $\overline{y y^{\prime}}$ is included in $D \cap B\left(y^{\prime}, 2 s\right)$, we find $y^{*} \in \partial^{*} D$ lying over $y^{\prime}$ such that $\rho_{D}\left(y, y^{*}\right)=\delta_{D}(y)<2 s$. Then $x, y \in B_{\rho}\left(y^{*}, 3 s\right)$. Observe from (7) that

$$
\begin{aligned}
& \rho_{D}\left(y^{*}, w\right) \geq \rho_{D}(y, w)-\rho_{D}\left(y, y^{*}\right) \geq\left(\frac{1}{c_{1}}-3\right) s=36 s \\
& \rho_{D}\left(y^{*}, z\right) \geq \rho_{D}(x, z)-\rho_{D}(x, y)-\rho_{D}\left(y, y^{*}\right) \geq\left(\frac{1}{c_{1}}-3\right) s=36 s,
\end{aligned}
$$

so that $w, z \in D \backslash B_{\rho}\left(y^{*}, 18 s\right)$ by (3). Lemma A implies

$$
\frac{G(y, w)}{G(x, w)} \approx \frac{G(y, z)}{G(x, z)},
$$


which together with (8) yields

$$
\frac{G(x, y) G(y, z)}{G(x, z)} \approx \frac{G(x, y) G(y, w)}{G(x, w)} \leq A \rho_{D}(x, y)^{2-d} .
$$

SubCASE 2b. $s=\rho_{D}(x, y) \leq \frac{1}{2} \delta_{D}(y)$. Then $G(x, y) \approx|x-y|^{2-d}=\rho_{D}(x, y)^{2-d}$ by Lemma 1. If furthermore $|y-z|>\frac{3}{4} \delta_{D}(y)$, then $G(\cdot, z)$ is positive and harmonic in $B\left(y, \frac{3}{4} \delta_{D}(y)\right)$, so that the Harnack inequality shows $G(x, z) \approx G(y, z)$ and hence

$$
\frac{G(x, y) G(y, z)}{G(x, z)} \approx G(x, y) \approx \rho_{D}(x, y)^{2-d} .
$$

If $|y-z| \leq \frac{3}{4} \delta_{D}(y)$, then $|y-z|=\rho_{D}(y, z) \geq \rho_{D}(x, y)=|x-y|$ by (6), so that $|x-z| \leq|x-y|+|y-z| \leq 2|y-z|$. Moreover, $G(y, z) \approx|y-z|^{2-d}$ and $G(x, z)$ $\approx|x-z|^{2-d}$; and hence,

$$
\frac{G(x, y) G(y, z)}{G(x, z)} \approx \frac{|x-y|^{2-d}|y-z|^{2-d}}{|x-z|^{2-d}} \leq A|x-y|^{2-d}=A \rho_{D}(x, y)^{2-d} .
$$

Thus (5) also holds in Case 2. The proof is complete.

\section{An example}

Let us begin with an application of the $3 \mathrm{G}$ inequality.

Proposition 1. Let $D$ be a domain of finite volume in $\mathbf{R}^{d}$ with $d \geq 3$. Suppose the $3 G$ inequality (1) holds. Then the following Cranston-McConnell inequality

$$
\sup _{x \in D} \frac{1}{u(x)} \int_{D} G(x, y) u(y) d y \leq d V_{d}^{1-2 / d}|D|^{2 / d} A_{0}
$$

holds for every nonnegative superharmonic function $u$ in $D$, where $V_{d}$ stands for the volume of the unit ball in $\mathbf{R}^{d}$.

Proof. Let $B(0, R)$ be the open ball with the same volume as $D$. Suppose $u$ is a Green potential $\int_{D} G(\cdot, z) d \mu(z)$ of a measure $\mu$ in $D$. Then (1) and Fubini's theorem yield

$$
\begin{aligned}
\int_{D} G(x, y) u(y) d y & =\int_{D} d \mu(z) \int_{D} G(x, y) G(y, z) d y \\
& \leq A_{0} \int_{D} G(x, z) d \mu(z) \int_{D}\left(|x-y|^{2-d}+|y-z|^{2-d}\right) d y \\
& \leq 2 A_{0} u(x) \int_{B(0, R)}|y|^{2-d} d y \\
& =A_{0} d V_{d} R^{2} u(x)=A_{0} d V_{d}^{1-2 / d}|D|^{2 / d} u(x)
\end{aligned}
$$


Thus (9) holds for a Green potential. Since every nonnegative superharmonic function is approximated from below by Green potentials, the monotone convergence theorem completes the proof.

For an arbitrary planar domain $D$ of finite area, Cranston and McConnell [14] proved (9) with $A_{0}$ bounded by the area of $D$ up to an absolute multiplicative constant. See [12] for a simple proof and $[1,5,2]$ for an analytic proof and some generalizations. Cranston and McConnell [14] provided also an example of bounded domain in $\mathbf{R}^{3}$, failing to satisfy (9). We shall modify their example to construct a bounded domain whose Martin boundary coincides with the Euclidean boundary and which fails to satisfy the Cranston-McConnell inequality (9). In view of Proposition 1, this domain also fails to satisfy the $3 \mathrm{G}$ inequality (1).

Construction. Let $R_{n} \downarrow 0$ and $N_{n} \uparrow \infty$ be a decreasing sequence of positive numbers and an increasing sequence of positive integers such that

(i) $R_{n+1}+\frac{R_{n+1}}{N_{n+1}} \leq R_{n}-\frac{R_{n}}{N_{n}}$,

(ii) $\sum_{n=1}^{\infty}\left(\frac{R_{n}}{N_{n}}\right)^{2} N_{n}^{d-1}=\infty$.

For example, $R_{n}=\frac{1}{\sqrt{n}}$ and $N_{n}=8 n$ satisfy the above condition. In fact,

$$
\begin{aligned}
R_{n}- & \frac{R_{n}}{N_{n}}-\left(R_{n+1}+\frac{R_{n+1}}{N_{n+1}}\right) \\
& \geq \frac{1}{\sqrt{n}}-\frac{1}{\sqrt{n+1}}-\frac{1}{4 n \sqrt{n}}=\frac{1}{\sqrt{n} \sqrt{n+1}(\sqrt{n}+\sqrt{n+1})}-\frac{1}{4 n \sqrt{n}} \\
& \geq \frac{1}{2(n+1) \sqrt{n}}-\frac{1}{4 n \sqrt{n}}=\frac{2 n-(n+1)}{4 n(n+1) \sqrt{n}}>0 ;
\end{aligned}
$$

and

$$
\left(\frac{R_{n}}{N_{n}}\right)^{2} N_{n}^{d-1}=\left(\frac{1}{8 n \sqrt{n}}\right)^{2}(8 n)^{d-1}=8^{d-3} n^{d-4} \geq 8^{d-3} n^{-1} .
$$

Let $0<\eta<1 / 4$ be a constant depending only on the dimension such that we can place $N_{n}^{d-1}$ many mutually disjoint open balls of radius $r_{n}=\eta R_{n} / N_{n}$ with centers on the sphere $S_{n}=\left\{x \in \mathbf{R}^{d}:|x|=R_{n}\right\}$. Order these balls and call them $B_{n}^{k}, k=1, \ldots, N_{n}^{d-1}$. In view of (i), we may assume that the family of the doubles of $B_{n}^{k}\left(n=1, \ldots, \infty\right.$ and $\left.k=1, \ldots, N_{n}^{d-1}\right)$ is mutually disjoint. Fix $n$ and connect each ball $B_{k}^{n}$ to the next $B_{k+1}^{n}$ for $k=1, \ldots, N_{n}^{d-1}-1$, in order, by a cylindrical tube lying in $B\left(0, R_{n}+2 r_{n}\right)$. Then connect the last ball $B_{n}^{k}$ with $k=N_{n}^{d-1}$ to the first ball $B_{n+1}^{1}$ of the $(n+1)$-th level by a cylindrical tube lying in $B\left(0, R_{n}+2 r_{n}\right)$. Moreover, each tube intersects its ball in circular caps 
subtending solid angle $\varepsilon<\pi / 6$ and the two caps in each ball (except the first) are antipodal. We may assume that the tubes are mutually disjoint and the connection is so smooth that the resultant domain $D$ is locally Lipschitz apart from the origin. Hence, we observe that the Martin boundary of $D$ is homeomorphic to the Euclidean boundary except for the origin. We shall show that there is a unique minimal function $h$ corresponding to the origin.

Proposition 2. Let $D$ be as above. Then there is a unique minimal function $h$ corresponding to the origin. Moreover,

$$
\int_{D} G(x, y) h(y) d y=\infty .
$$

Hence, the Martin boundary of D coincides with the Euclidean boundary and yet the Cranston-McConnell inequality (9) and the $3 G$ inequality (1) fail to hold.

We prepare the proof of Proposition 2 by stating the following boundary Harnack principle for a specific Lipschitz domain. Since we consider near a smooth boundary portion, the boundary Harnack principle can be proved easily. See Figure 1.

LEMMA 2. Let $\Omega=\left\{x=\left(x_{1}, \ldots, x_{d}\right): \frac{1}{4}<|x|<1,-\frac{\sqrt{3}}{2}<x_{1}<0\right\}, \quad H=$ $\left\{x \in \Omega: x_{1}=-\frac{1}{2}\right\}$ and $x^{*}=\left(-\frac{1}{2}, 0, \ldots, 0\right)$. If $u$ and $v$ are positive harmonic functions on $\Omega$ such that $u=v=0$ on $\left\{x=\left(x_{1}, \ldots, x_{d}\right):|x|=1,-\frac{\sqrt{3}}{2}<x_{1}<0\right\}$, then

$$
\frac{u(x)}{u\left(x^{*}\right)} \approx \frac{v(x)}{v\left(x^{*}\right)} \approx \frac{\delta_{\Omega}(x)}{\delta_{\Omega}\left(x^{*}\right)} \quad \text { for } x \in H
$$

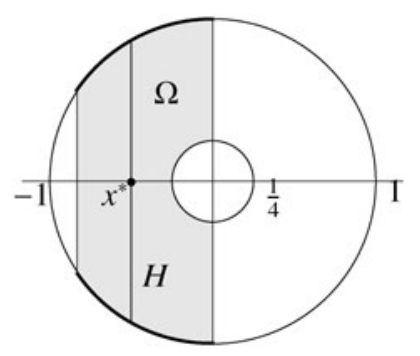

FIGURE 1. Boundary Harnack Principle for the shaded domain $\Omega$.

Proof of Proposition 2. Let $B_{0}=B\left(x_{0}, \rho_{0}\right), \quad B_{1}=B\left(x_{1}, \rho_{1}\right), \ldots$ be the enumeration of $\left\{B_{n}^{k}\right\}_{n, k}$ in order and let $T_{j}$ be the tube connecting $B_{j}$ and $B_{j+1}$. Our domain $D$ looks like a long wiggling string of beads. Take $j \geq 1$. We may assume by rotation that $B_{j}$ and $T_{j}$ intersect in a circular cap with center at $\left(-\rho_{j}, 0, \ldots, 0\right)+x_{j}$. Translate and dilate $\Omega$ in Lemma 2 so that $x_{j}$ and 
$\left(-\rho_{j}, 0, \ldots, 0\right)+x_{j}$ correspond to the origin and $(-1,0, \ldots, 0)$, respectively. Let $H_{j}$ and $x_{j}^{*}$ correspond to $H$ and $x^{*}$, respectively. Observe that $B_{j} \backslash H_{j}$ consists of two connected components. By $B_{j}^{\prime}$ we denote the component containing $x_{j}$. Let $L_{j}=B_{0} \cup T_{0} \cup B_{1} \cup T_{1} \cup \cdots \cup T_{j-1} \cup B_{j}^{\prime}$ and let $U_{j}=D \backslash\left(L_{j} \cup H_{j}\right)$. See Figure 2 .

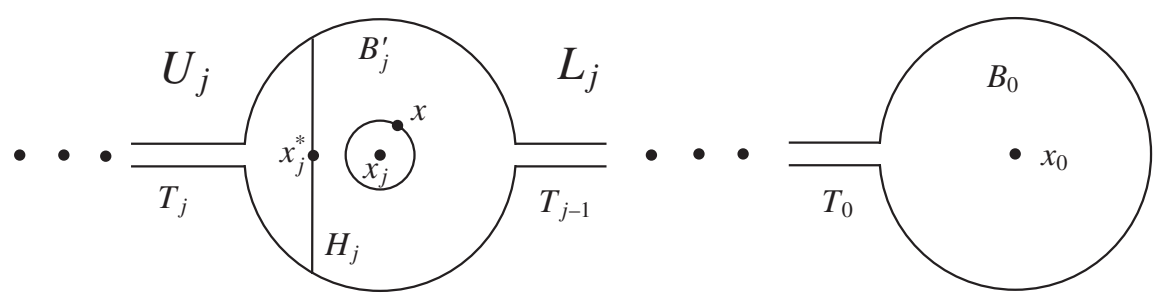

FIgURE 2. Counter example to the Cranston-McConnell inequality: a long wiggling string of beads.

Fix $x$ such that $\left|x-x_{j}\right|=\rho_{j} / 4$. Apply Lemma 2 to $u=G(x, \cdot)$ and $v=$ $G\left(x_{0}, \cdot\right)$. Then

$$
\frac{G(x, y)}{G\left(x, x_{j}^{*}\right)} \approx \frac{G\left(x_{0}, y\right)}{G\left(x_{0}, x_{j}^{*}\right)} \quad \text { for } y \in H_{j} \text { and hence for } y \in U_{j}
$$

by the maximum principle. Since $G\left(x, x_{j}^{*}\right) \approx \rho_{j}^{2-d}$, it follows that

$$
\frac{G(x, y)}{G\left(x_{0}, y\right)} \approx \frac{\rho_{j}^{2-d}}{G\left(x_{0}, x_{j}^{*}\right)} \text { for } y \in U_{j}
$$

Let $K(x, y)=G(x, y) / G\left(x_{0}, y\right)$ for $x \in D$ and $y \in D \backslash\left\{x_{0}\right\}$. The Martin kernel is given as the limit of $K(x, y)$ when $y$ tends to a boundary point. Let $u$ and $v$ be Martin kernels at 0 with respect to $x_{0}$. Then the above estimate implies

$$
u(x) \approx v(x) \approx \frac{\rho_{j}^{2-d}}{G\left(x_{0}, x_{j}^{*}\right)} \text { for }\left|x-x_{j}\right|=\rho_{j} / 4 \text { and hence for }\left|x-x_{j}\right| \leq \rho_{j} / 4
$$

by the maximum principle. By the Harnack inequality

$$
u\left(x_{j}^{*}\right) \approx v\left(x_{j}^{*}\right) \approx \frac{\rho_{j}^{2-d}}{G\left(x_{0}, x_{j}^{*}\right)},
$$

so that the boundary Harnack principle (Lemma 2) gives a constant $A_{3}>1$ such that

$$
A_{3}^{-1} u(x) \leq v(x) \leq A_{3} u(x)
$$

for $x \in H_{j}$ and hence for $x \in L_{j}$ by the maximum principle. Since $j$ is arbitrary, we have (11) for all $x \in D$.

Now, a standard technique ([3, Theorem 3]) shows that there exists a unique minimal Martin kernel at 0. For the reader's convenience we give a proof. Let 
$\mathscr{H}_{0}$ be the family of all positive harmonic functions $u$ on $D$ vanishing on $\partial D \backslash\{0\}$, bounded on $D \backslash B(0, r)$ for each $r>0$ and taking value $u\left(x_{0}\right)=1$. Obviously, a Martin kernel at 0 belongs to $\mathscr{H}_{0}$. Since every $u \in \mathscr{H}_{0}$ can be represented as an integral over Martin kernels at 0 , we see that (11) extends to $u, v \in \mathscr{H}_{0}$. Let

$$
c=\sup _{\substack{u, v \in \mathscr{H}_{0} \\ x \in D}} \frac{u(x)}{v(x)}
$$

Then $1 \leq c \leq A_{3}^{2}<\infty$ by (11). Let us show that $c=1$. Suppose to the contrary $c>1$. Take arbitrary $u, v \in \mathscr{H}_{0}$. Then $v_{1}=(c v-u) /(c-1) \in \mathscr{H}_{0}$, so that $u \leq c v_{1}=c(c v-u) /(c-1)$ by (12). Hence $(2 c-1) u \leq c^{2} v$ on $D$, which would imply

$$
c=\sup _{\substack{u, v \in \mathscr{H} \\ x \in D}} \frac{u(x)}{v(x)} \leq \frac{c^{2}}{2 c-1}<c,
$$

a contradiction. Thus $c=1$ and $\mathscr{H}_{0}$ is a singleton consisting of the Martin kernel $K(\cdot, 0)$ at 0 . Moreover, the Martin kernel $K(\cdot, 0)$ is minimal since there is at least one minimal Martin kernel at 0 .

Let $h=K(\cdot, 0)$ be the Martin kernel at 0 . Then (10) and the Harnack inequality give

$$
\int_{B\left(x_{j}, \rho_{j} / 4\right)} G\left(x_{0}, y\right) h(y) d y \approx G\left(x_{0}, x_{j}^{*}\right) \frac{\rho_{j}^{2-d}}{G\left(x_{0}, x_{j}^{*}\right)} \rho_{j}^{d} \approx \rho_{j}^{2} .
$$

In view of Construction (ii), we obtain

$$
\int_{D} G\left(x_{0}, y\right) h(y) d y \geq \sum_{j=1}^{\infty} \int_{B\left(x_{j}, \rho_{j} / 4\right)} G\left(x_{0}, y\right) h(y) d y=\infty .
$$

By the Harnack inequality the above integral diverges for every $x \in D$ in place of $x_{0}$ as well. The proof is complete.

\section{REFERENCES}

[1] H. Aikawa, On the upper bounds of Green potentials, Hiroshima Math. J. 24 (1994), 607612.

[2] H. Aikawa, Generalized Cranston-McConnell inequalities for discontinuous superharmonic functions, Potential Anal. 8 (1998), 127-135.

[ 3 ] H. Aikawa, Boundary Harnack principle and Martin boundary for a uniform domain, J. Math. Soc. Japan 53 (2001), 119-145.

[4] H. Aikawa, T. Lundh and T. Mizutani, Martin boundary of a fractal domain, Potential Anal. 18 (2003), 311-357.

[5] H. Aikawa and M. Murata, Generalized Cranston-McConnell inequalities and Martin boundaries of unbounded domains, J. Anal. Math. 69 (1996), 137-152. 
[6] A. AnConA, Principe de Harnack à la frontière et théorème de Fatou pour un opérateur elliptique dans un domaine lipschitzien, Ann. Inst. Fourier (Grenoble) 28-4 (1978), 169-213.

[ 7 ] Z. BALOGH AND A. Volberg, Boundary Harnack principle for separated semihyperbolic repellers, harmonic measure applications, Rev. Mat. Iberoamericana 12 (1996), 299-336.

[8] Z. BAlOGH AND A. Volberg, Geometric localization, uniformly John property and separated semihyperbolic dynamics, Ark. Mat. 34 (1996), 21-49.

[9] R. F. BASs, Probabilistic techniques in analysis, Springer-Verlag, New York, 1995, Correction on http://www.math.uconn.edu/ bass.

[10] R. F. BASS AND K. Burdzy, Conditioned Brownian motion in planar domains, Probab. Theory Related Fields 101 (1995), 479-493.

[11] M. Bonk, J. Heinonen and P. Koskela, Uniformizing Gromov hyperbolic spaces, Astérisque 270 (2001), viii+99.

[12] K. L. Chung, The lifetime of conditional Brownian motion in the plane, Ann. Inst. H. Poincaré Probab. Statist. 20 (1984), 349-351.

[13] M. Cranston, E. Fabes and Z. Zhao, Conditional gauge and potential theory for the Schrödinger operator, Trans. Amer. Math. Soc. 307 (1988), 171-194.

[14] M. Cranston and T. R. McConnell, The lifetime of conditioned Brownian motion, $\mathrm{Z}$. Wahrsch. Verw. Gebiete 65 (1983), 1-11.

[15] B. E. J. Dahlberg, Estimates of harmonic measure, Arch. Rational Mech. Anal. 65 (1977), $275-288$.

[16] R. A. Hunt And R. L. Wheeden, Positive harmonic functions on Lipschitz domains, Trans. Amer. Math. Soc. 147 (1970), 507-527.

[17] J. VÄıs̈̈LÄ, Relatively and inner uniform domains, Conform. Geom. Dyn. 2 (1998), 56-88 (electronic).

[18] J. M. G. WU, Comparisons of kernel functions, boundary Harnack principle and relative Fatou theorem on Lipschitz domains, Ann. Inst. Fourier (Grenoble) 28-4 (1978), 147-167.

Note added in proof.

Wolfhard Hansen informed the authors that he wrote related papers:

(i) Global comparison of perturbed Green functions, to appear in Math. Ann.

(ii) Uniform boundary Harnack principle and generalized triangle property, to appear in J. Funct. Anal.

(iii) Simple counterexamples to the 3G-inequality, to appear in Expo. Math.

In particular, (iii) gave another simple example corresponding to that in Section 3 of this paper.

\author{
Department of Mathematics \\ SHIMANE UNIVERSITY \\ MATSUE 690-8504 \\ JAPAN \\ E-mail address: haikawa@riko.shimane-u.ac.jp \\ Department of Mathematics \\ Chalmers University of Technology \\ S-412 96 GÖTEBORG \\ SwEDEN \\ E-mail address: torbjrn@math.chalmers.se
}

